\title{
Comparison of bypass surgery and drug-eluting stenting in diabetic patients with left main and/or multivessel disease: A systematic review and meta-analysis of randomized and nonrandomized studies
}

\author{
Fumei Huang ${ }^{1}$, Wenyuan Lai ${ }^{1}$, Choileng Chan ${ }^{1}$, Hui Peng ${ }^{2}$,
} Feifei Zhang ${ }^{1}$, Yi Zhou ${ }^{3}$, Shuen Teng ${ }^{1}$, Zheng Huang ${ }^{1}$

${ }^{1}$ Department of Cardiology, Nanfang Hospital, Southern Medical University, Guangzhou, Guangdong, China

${ }^{2}$ Department of Sleep Disorder Center, Nanfang Hospital, Southern Medical University,

Guangzhou, Guangdong, China

${ }^{3}$ Department of Obstetrics and Gynecology, Nanfang Hospital, Southern Medical University,

Guangzhou, Guangdong, China

Abstract

Background: With advances in theinterventional field, the choice between coronary artery bypass grafting $(C A B G)$ and percutaneous coronary intervention with drug-eluting stents (PCI-DES) for the diabetic subset with left main (LM) and/or multivessel disease (MVD) remains consistently controversial.

Methods and results: We conducted a systematic review of randomized controlled trials (RCTs) and observational controlled trials (OCTs) comparing the two strategies for the diabetic subset with LM and/or MVD. PubMed, EMBASE, CENTRAL databases, Google Scholar and SinoMed were systematically searched for eligible studies without language and publication restrictions. We identified 19 trials (4 randomized and 15 nonrandomized), enrolling 5,805 patients in OCTs and 3,060 patients in RCTs, respectively. PCI-DES was associated with higher mortality compared with CABG (11.7\% DES vs. 9.1\% CABG, RR 1.23, 95\% CI $1.00-1.53, p=0.06$ ). Patients after PCI-DES had higher prevalence of myocardial infarction (MI) when compared with CABG (8.5\% DES vs. 4.6\% CABG, RR 1.68, 95\% CI 1.20-2.37, $p=0.003) . P C I-D E S$ patients were at substantially lower risk of stroke $(2.0 \%$ DES vs. $3.9 \%$ $C A B G, R R$ 0.51, 95\% CI 0.39-0.67, p < 0.00001), but at several-fold higher risk of repeat revascularization (19.0\% DES vs. $6.3 \%$ CABG, RR 2.95, 95\% CI 2.46-3.55, $p<0.00001$ ). The OCT patients risked a lower mortality as compared to the RCT patients $(9.6 \%$ OCTs vs. $11.9 \%$ RCTs, RR 0.81, 95\% CI 0.71-0.92, $p=0.001$ ).

Conclusions: $C A B G$ for patients with diabetes mellitus and LM and/or MVD had advantages over PCI-DES in all-cause death, nonfatal MI, and repeat revascularization, but the substantial disadvantage in nonfatal stroke. The high-selected patients (RCTs) risked a higher mortality than the real-world patients (OCTs). (Cardiol J 2015; 22, 2: 123-134)

Key words: coronary artery bypass grafting, multivessel disease, left main, diabetes mellitus, drug-eluting stents

Address for correspondence: Zheng Huang, MD, PhD, Department of Cardiology, Nanfang Hospital, Southern Medical University, 1838 North Guangzhou Avenue, Guangzhou, Guangdong, China, 510515, e-mail: drhuangzheng@163.com 


\section{Introduction}

Diabetes mellitus is a powerful, independent risk factor for cardiovascular disease and accounts for about $25 \%$ of all patients requiring myocardial revascularization $[1,2]$. Patients with diabetes have more extensive and diffuse coronary artery disease (CAD) than nondiabetic patients [3, 4], and have higher mortality and morbidity after revascularization procedures, including myocardial infarction (MI), restenosis after balloon angioplasty [4-7], and bare-metal stenting [8]. Despite significant improvements in the CAD mortalities in the past decades, due to atherosclerosis resulting in MI, CAD remains the leading death death worldwide [5]. Coronary artery bypass grafting (CABG) was generally regarded as a preferred revascularization strategy for patients with left main (LM) and/or multivessel disease (MVD) [9]. However, the advances in the interventional field, especially the advent and development of drug-eluting stents (DES), which significantly reduced restenosis and the need for subsequent repeat revascularizations as compared with bare metal stents (BMS), have largely cut back one of the major limitations of percutaneous coronary interventions (PCI) [10-18]. Several randomized controlled trials (RCTs), exclusively comparing CABG surgery and PCI with DES (PCI-DES) for the diabetic subset with LM and/or MVD [19-22], have reported medium- and long-term outcomes, but given high-selected patients in the RCTs, their applicability to the general population is unknown. The observational controlled trial (OCTs), unlike the RCTs, can reflect daily clinical practice in the real world. We conducted a systematic review and meta-analysis of randomized and nonrandomized studies to establish clinical efficacy and safety of PCI-DES vs. CABG in patients with diabetes and LM and/or MVD both in the real world (OCTs) and in high-selected population (RCTs).

\section{Methods}

\section{Search strategy}

We systematically searched PubMed, EMBASE, the Cochrane Central Register of Controlled Trials, Google Scholar and SinoMed for relevant studies reported from January 2002 (the year when the DESs were introduced to clinical practice) to December 2013, without language and publication restrictions. To achieve the maximum sensitivity of the search strategies and identify all trials comparing PCI-DES with CABG in diabetic subset, we appropriately used both free text and thesaurus terms, including: 'multivessel disease', 'left main', 'diabetes mellitus', 'percutaneous coronary intervention', 'drug-eluting stents', and 'coronary artery bypass'. We also performed a systematic search from reference lists of selected articles, conference proceedings, and personal files for relevant citations.

\section{Inclusion and exclusion criteria}

Studies were included in this meta-analysis if they met the following criteria: 1) RCT, OCT and pre-specified subgroup analyses comparing CABG with PCI-DES for diabetics with LM and/or MVD; 2) studies published in peer-reviewed journals with full available text; and 3) follow-up period $\geq 12$ months. Studies were excluded if they met any of the following criteria: 1 ) the subjects were not exclusively diabetics with LM and/or MVD, 2) using only BMS or involving BMS with DES in $1 \mathrm{PCI}$ subject, 3) duplicate publication, 4) less than 50 patients in each cohort.

\section{Study selection}

Two reviewers screened the citations and abstracts identified by the search strategies. Full text reviews were also conducted by 2 other reviewers to establish eligibility when screening reviewers believed that a citation potentially met the inclusion criteria. Disagreements regarding inclusion were resolved via consensus.

\section{Data extraction}

Three reviewers independently extracted data from the eligible studies. The following information were extracted from each study: first author, year of publication, duration of follow-up, number of participants in each group (CABG or PCI-DES), baseline characteristics, and outcome events including: all-cause mortality (the primary outcome), non-fatal MI, non-fatal stroke and repeat revascularization (Table 1). Each OCT was named by the family name plus the publication year (family name + year) respectively, and the RCT was presented as its own study name. For studies reported in $>1$ publications, we extracted data from the most complete publication and used other publications as supplements. We also tried our best to contact the authors by email for information if their articles did not report the information in detail.

\section{Statistical methods}

Data were summarized using descriptive statistics. Discrete variables were presented as proportions (\%, count/sample size) and compared 
Table 1. Baseline characteristics of clinical studies.

\begin{tabular}{|c|c|c|c|c|c|c|}
\hline Study or subgroup & $\begin{array}{l}\text { Location or } \\
\text { centers (n) }\end{array}$ & $\begin{array}{l}\text { Subjects (n) } \\
\text { (DES/CABG) }\end{array}$ & Design & Follow-up & $\begin{array}{c}\text { Type of revas- } \\
\text { cularization }\end{array}$ & $\begin{array}{l}\text { Coronary } \\
\text { lesion }\end{array}$ \\
\hline Ben-Gal, 2006 [23] & Israel & $86 / 86$ & OCT & 18 months & DES/CABG & $\begin{array}{c}\text { Single, } \\
\text { multivessel } \\
\text { and/or } \\
\text { LM disease }\end{array}$ \\
\hline Briguori, 2007 [24] & Italy & $69 / 149$ & OCT & 1 year & DES/OPCABG & $\begin{array}{l}\text { MVD and critical } \\
\text { stenosis involv- } \\
\text { ing proximal LAD }\end{array}$ \\
\hline Contini, 2012 [25] & Italy & $359 / 359$ & OCT & 5 years & DES/CABG & MVD \\
\hline $\begin{array}{l}\text { Domínguez-Franco, } \\
2008 \text { [26] }\end{array}$ & Spain & $128 / 142$ & OCT & 24 months & DES/CABG & $\begin{array}{c}\text { MVD } \\
(\geq 2 \text { vessels } \\
\text { with a }>70 \% \\
\text { de novo stenosis } \\
\text { involving the } \\
\text { proximal LAD) }\end{array}$ \\
\hline Javaid, 2007 [27] & United States & $344 / 257^{\ddagger}$ & OCT & 12 months & DES/CABG & MVD \\
\hline Kim, 2009 [28] & 12 & $251 / 256$ & OCT & 3 years & $\begin{array}{l}\text { SES, PES/ } \\
\text { /CABG }\end{array}$ & $\begin{array}{c}\text { Unprotected } \\
\text { LMCAD } \\
\text { (defined as } \\
\text { stenosis } \geq 50 \% \text { ) }\end{array}$ \\
\hline Lee, 2007 [29] & United States & $102 / 103$ & ОСТ & 1 year & DES/CABG & MVD \\
\hline Luo, 2012 [30] & China & $99 / 127$ & OCT & 25.3 months & DES/CABG & $\begin{array}{c}\text { Unprotected } \\
\text { LMCAD } \\
\text { (defined as } \\
\text { stenosis } \geq 50 \% \text { ) }\end{array}$ \\
\hline Moshkovitz, 2012 [31] & Israel & $271 / 226$ & ОСТ & 62 months & DES/BITA & MVD \\
\hline Onuma, 2011 [32] & Netherlands & $159 / 96$ & ОСТ & 5 years & SES/CABG & MVD \\
\hline Park, $2011[33,34]$ & Korea & $489 / 402$ & OCT§ & 5.6 years & DES/CABG & MVD \\
\hline Qiao, 2009 [35] & China & $363 / 282$ & OCT & 12 months & DES/CABG & MVD \\
\hline Tarantini, 2009 [36] & Italy & $93 / 127$ & ОСТ & 2 years & DES/CABG & MVD \\
\hline Yamagata, 2010 [37] & Japan & $92 / 116$ & Ост & 42 months & SES/OPCAB & MVD \\
\hline Zhao, 2011 [38] & China & $56 / 116$ & OCT & $\begin{array}{l}28.5 \text { months } \\
\text { DES; } \\
28.4 \text { months } \\
\text { CABG }\end{array}$ & DES/CABG & LMCAD \\
\hline CARDia [22] & 24 & $256 / 254$ & $\mathrm{RCT}$ & 5.1 years & $\begin{array}{l}\text { BMS, DES/ } \\
\text { /CABG* }\end{array}$ & $\begin{array}{c}\text { MVD or complex } \\
\text { single-vessel } \\
\text { CAD }\end{array}$ \\
\hline FREEDOM [21] & 140 & $953 / 947$ & $\mathrm{RCT}$ & 3.8 years & $\begin{array}{l}\text { SES, PES/ } \\
\text { /CABG }\end{array}$ & MVD \\
\hline SYNTAX [20] & 85 & $231 / 221$ & $\mathrm{RCT \S}$ & 5 years & PES/CABG & $\begin{array}{l}\text { LM (isolated or in } \\
\text { addition to } \\
1-, 2-, \text { or } 3 \text {-vessel } \\
\text { disease) or } \\
\text { isolated } 3 \text {-vessel } \\
\text { disease }\end{array}$ \\
\hline VA CARDS [19] & 22 & $101 / 97$ & RCT & 2 years & DES/CABG & Severe CAD \\
\hline
\end{tabular}

by the $\chi^{2}$ test or Fisher's exact test, where appropriate. Continuous variables were presented as mean \pm standard deviation and compared using the Student's t-test. The verified data were ana- lyzed using Revman software (version 5.2). The endpoints of each study were analyzed using risk ratio (RR) with $95 \%$ confidence interval (CI). The Cochrane $\chi^{2}$ (Cochrane $\mathrm{Q}$ ) test was used to assess 


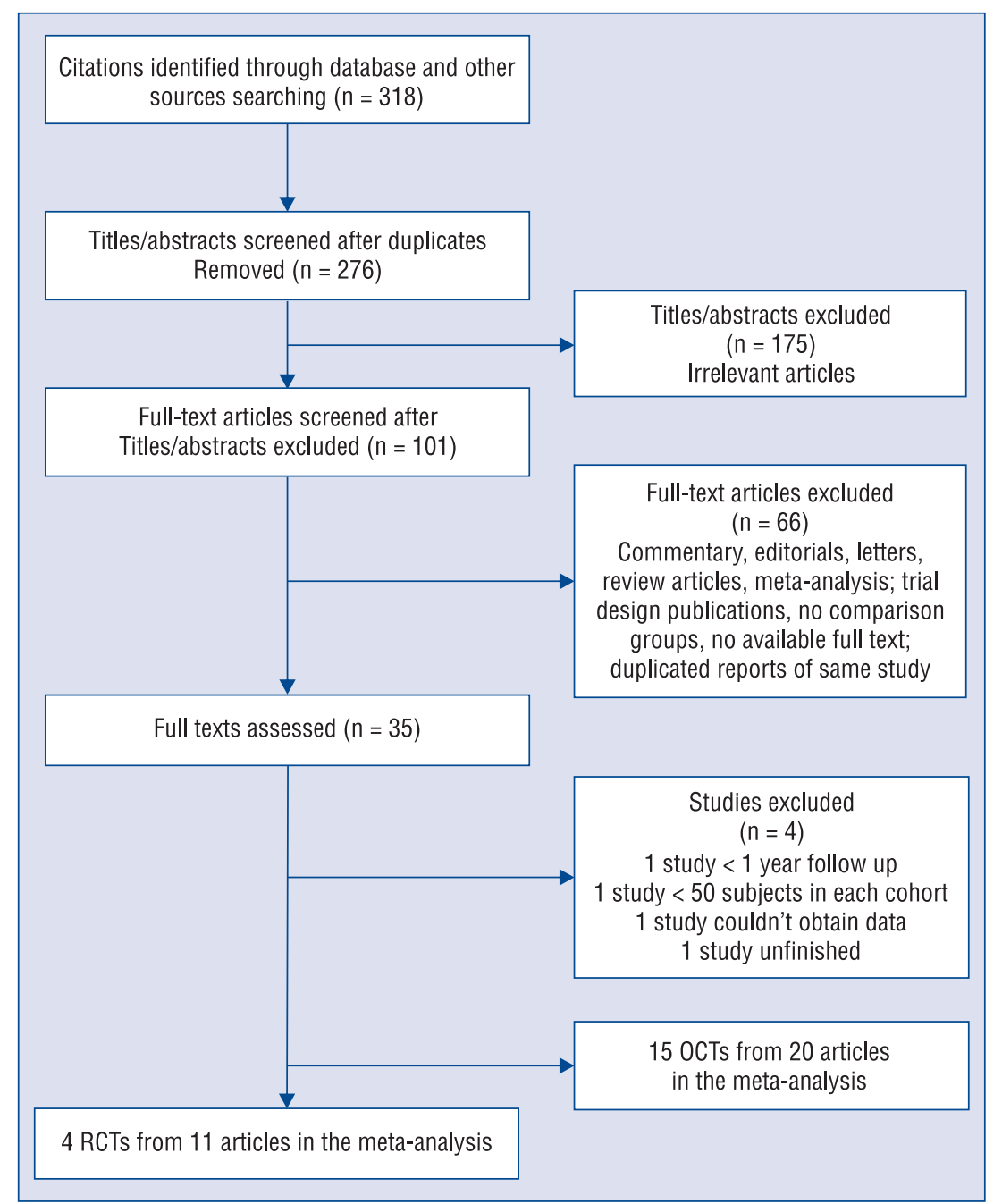

Figure 1. Flow diagram for inclusion of studies; OCTs - observational controlled trials; RCTs — randomized controlled trials.

the between-trial heterogeneity. The $\mathrm{I}^{2}$ statistic was calculated as a measure of the proportion of the overall variation attributable to the betweentrial heterogeneity rather than to chance, and we used the reported guidelines for low $\left(\mathrm{I}^{2}=25-49 \%\right)$, moderate $\left(\mathrm{I}^{2}=50-74 \%\right)$, and high $\left(\mathrm{I}^{2} \geq 75 \%\right)$ heterogeneity [39]. The overall effect size (RR) was calculated by fixed-effect model with the Mantel-Haenszel method when there was no significant heterogeneity $\left(\mathrm{p}>0.10\right.$ or $\left.\mathrm{I}^{2}<50 \%\right)$, or with DerSimonian-Laird weights for the random-effects model when there was a significant heterogeneity $\left(\mathrm{p} \leq 0.10\right.$ or $\left.\mathrm{I}^{2} \geq 50 \%\right)$. Forest plots were then created for graphical presentations of clinical outcomes. Publication bias with respect to the primary outcome (all-cause death) was assessed visually using a funnel plot. When there is no publication bias, studies of all sizes are scattered equally right and left of the line indicating the pooled estimate of natural log RR. For each endpoint we conducted subgroup analyses in RCTs and OCTs respectively, apart from an overall analysis. A sensitivity analysis was performed when the between-trial heterogeneity was significant.

\section{Results}

We identified 19 eligible studies (4 randomized [19-22] and 15 nonrandomized [23-38]) (Fig. 1) enrolling 5,805 patients in OCT subgroup (PCI-DES: 2,961; CABG: 2,844) and 3,060 in RCT subgroup (PCI-DES: 1,541; CABG: 1,519), respectively. The mean follow-up durations ranged from 1 year to 5.6 years. In the OCTs, with the exception of the propensity score-matched studies $[23,25]$, the CABG cohorts had higher prevalence of the triple vessel and/or LM disease, higher EuroSCORE and/or higher SYNTAX Score, but not in the RCTs (Table 2). 


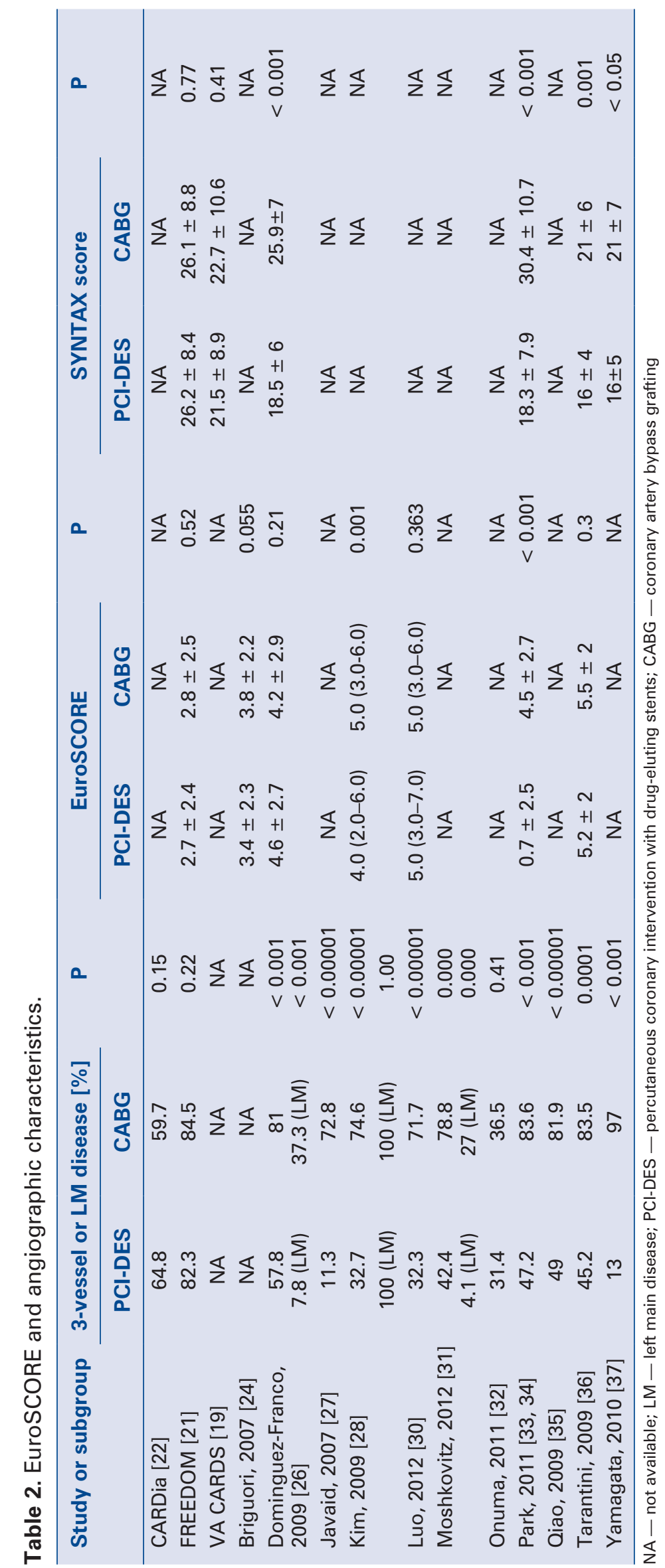




\begin{tabular}{|c|c|c|c|c|c|c|c|c|}
\hline \multirow[b]{2}{*}{ Studv or Subgroup } & \multicolumn{2}{|c|}{ PCI-DES } & \multicolumn{2}{|c|}{ CABG } & \multirow{2}{*}{ Weight } & \multirow{2}{*}{$\begin{array}{c}\text { Risk Ratio } \\
\text { M-H, Random, } 95 \% \mathrm{Cl}\end{array}$} & \multirow{2}{*}{\multicolumn{2}{|c|}{$\begin{array}{c}\text { Risk Ratio } \\
\text { M-H, Random, } 95 \% \mathrm{Cl}\end{array}$}} \\
\hline & Events & Total & Events & Total & & & & \\
\hline \multicolumn{9}{|l|}{ 1.1.1 OCTs } \\
\hline Ben-Gal2006 & 2 & 86 & 3 & 86 & $1.3 \%$ & $0.67[0.11,3.89]$ & & \\
\hline Briguori200? & 4 & 69 & $?$ & 149 & $2.6 \%$ & $1.23[0.37,4.08]$ & & \\
\hline Contini2012 & 88 & 359 & 65 & 359 & $11.5 \%$ & $1.35[1.02,1.80]$ & & $=$ \\
\hline Domínguez-Franco2008 & 11 & 124 & 13 & 139 & $5.1 \%$ & $0.95[0.44,2.04]$ & - & - \\
\hline Javaid2007 & 46 & 344 & 7 & 257 & $5.0 \%$ & $4.91[2.25,10.69]$ & & \\
\hline Kim2009 & 16 & 251 & 22 & 256 & $6.5 \%$ & $0.74[0.40,1.38]$ & $\rightarrow$ & T \\
\hline Lee2007 & 10 & 102 & 8 & 103 & $4.2 \%$ & $1.26[0.52,3.07]$ & - & - \\
\hline Luo2012 & 6 & 99 & 11 & 127 & $3.7 \%$ & $0.70[0.27,1.83]$ & $\longrightarrow$ & - \\
\hline Onuma2011 & 14 & 159 & 8 & 96 & $4.6 \%$ & $1.06[0.46,2.43]$ & - & - \\
\hline Park2011 & 57 & 489 & 60 & 402 & $10.6 \%$ & $0.78[0.56,1.09]$ & $\rightarrow$ & \\
\hline Qiao2009 & 11 & 363 & 9 & 282 & $4.3 \%$ & $0.95[0.40,2.26]$ & & — \\
\hline Tarantini2008 & 4 & 93 & 5 & 127 & $2.3 \%$ & $1.09[0.30,3.96]$ & & \\
\hline Yamagata2010 & 5 & 92 & 5 & 116 & $2.6 \%$ & $1.26[0.38,4.22]$ & & \\
\hline Zhao 2011 & 3 & 55 & 7 & 108 & $2.3 \%$ & $0.84[0.23,3.13]$ & & 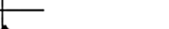 \\
\hline Subtotal $(95 \% \mathrm{Cl})$ & & 2685 & & 2607 & $66.6 \%$ & $1.11[0.84,1.45]$ & & \\
\hline Total events & 277 & & 230 & & & & & \\
\hline \multirow{2}{*}{\multicolumn{9}{|c|}{$\begin{array}{l}\text { Heterogeneity: } \text { Tau }^{2}=0.10 ; \mathrm{Chi}^{2}=23.70, \mathrm{df}=13(P=0.03) ; \mathrm{I}^{2}=45 \% \\
\text { Test for overall effect: } Z=0.72(P=0.47)\end{array}$}} \\
\hline & & & & & & & & \\
\hline \multicolumn{9}{|l|}{ 1.1.2 RCTs } \\
\hline CARDia & 37 & 254 & 32 & 248 & $9.0 \%$ & $1.13[0.73,1.75]$ & & E \\
\hline FREEDOM & 114 & 953 & 83 & 947 & $11.7 \%$ & $1.36[1.04,1.78]$ & & - \\
\hline SYNTAX & 44 & 231 & 26 & 221 & $8.8 \%$ & $1.62[1.03,2.54]$ & & $\rightarrow$ \\
\hline VA CARDS & 21 & 101 & 5 & 97 & $3.9 \%$ & $4.03[1.58,10.27]$ & & $\longrightarrow$ \\
\hline Subtotal $(95 \% \mathrm{Cl})$ & & 1539 & & 1513 & $33.4 \%$ & $1.51[1.09,2.10]$ & & $\bullet$ \\
\hline Total events & 216 & & 146 & & & & & \\
\hline \multirow{2}{*}{\multicolumn{9}{|c|}{$\begin{array}{l}\text { Heterogeneity: } \mathrm{Tau}^{2}=0.06 ; \mathrm{Ch}^{2}=6.28, \mathrm{df}=3(\mathrm{P}=0.10) ; \mathrm{I}^{2}=52 \% \\
\text { Test for overall effect: } Z=2.47(\mathrm{P}=0.01)\end{array}$}} \\
\hline & & & & & & & & \\
\hline Total $(95 \% \mathrm{Cl})$ & & 4224 & & 4120 & $100.0 \%$ & $1.23[1.00,1.53]$ & & 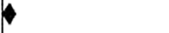 \\
\hline \multirow{3}{*}{\multicolumn{7}{|c|}{$\begin{array}{l}\text { Heterogeneity: } \text { Tau}^{2}=0.08 ; \mathrm{Chi}^{2}=33.73, \mathrm{df}=17(\mathrm{P}=0.009) ; \mathrm{l}^{2}=50 \% \\
\text { Test for overall effect: } Z=1.92(\mathrm{P}=0.06)\end{array}$}} & & \\
\hline & & & & & & & $0.01 \quad 0.1$ & 10 \\
\hline & & & & & & & $\begin{array}{l}\text { 0.0T } 0.1 \\
\text { Favours PCI-DES }\end{array}$ & Favours $C A B G$ \\
\hline \multicolumn{7}{|c|}{$\begin{array}{l}\text { Test for overall effect: } Z=1.92(P=0.06) \\
\text { Test for subaroun differences: } \mathrm{Chi}^{2}=2.04 . \mathrm{df}=1(\mathrm{P}=0.15) . \mathrm{I}^{2}=51.1 \%\end{array}$} & & \\
\hline
\end{tabular}

Figure 2. All-cause mortality at longest follow-up. The individual trials and subtotal risk ratios and $95 \%$ confidence intervals $(\mathrm{Cl})$ comparing the outcome of death for percutaneous coronary intervention with drug-eluting stents (PCI-DES) vs. coronary artery bypass grafting (CABG).

The CARDia trial included patients initially undergoing PCI with BMS (BMS 31\%, DES 69\%) [22]. The VA CARDS trial was stopped because of slow recruitment after enrolling only $25 \%$ of the intended sample size, leaving it severely underpowered for the primary composite endpoint of death plus nonfatal MI [19].

\section{All-cause death}

Random-effects meta-analysis yielded two different outcomes for all-cause death in RCTs and OCTs subgroup. The prevalence of death in RCTs subgroup was $14.0 \%$ in the PCI-DES cohort and $9.6 \%$ in CABG cohort with an RR of 1.51 (95\% CI 1.09-2.10, $\mathrm{p}=0.01$ ). A moderate heterogeneity among the RCTs, mainly driven by including the VA CARDS trial, was revealed by sensitivity analysis $\left(\mathrm{I}^{2}=52 \%, \mathrm{p}=0.10\right)$. After excluding VA CARDS trial, random-effects model generated an RR 1.36, 95\% CI 1.11-1.66 for death $(\mathrm{p}=0.003)$ with no residual heterogeneity $\left(\mathrm{I}^{2}=0 \%, \mathrm{p}=0.53\right)$. While the OCT subgroup analysis indicated a comparable mortality between PCI-DES and CABG (10.3\% DES vs. $8.8 \%$ CABG, RR $1.08,95 \%$ CI $0.83-1.42$, $\mathrm{p}=0.55$ ), heterogeneity analysis revealed a low het- erogeneity among the OCTs $\left(\mathrm{I}^{2}=45 \%, \mathrm{p}=0.03\right)$ which was largely due to the inclusion of the Javaid, 2007 trial. After the exclusion of this trial, random-effects meta-analysis yielded an RR 1.02 (95\% CI $0.85-1.22)$ for death $(\mathrm{p}=0.80)$ with no residual heterogeneity $\left(\mathrm{I}^{2}=0 \%, \mathrm{p}=0.73\right)$.

When pooling all the RCTs and OCTs, random-effects model yielded an RR 1.23 (11.7\% DES vs. 9.1\% CABG, 95\% CI 1.00-1.53, $\mathrm{p}=0.06$ ) for death. After excluding the VA CARDS trial and the Javaid, 2007 trial, the overall mortalities of the two arms reached statistical difference (RR 1.15, 95\% CI $1.01-1.31, \mathrm{p}=0.04)$ with no residual heterogeneity between trials $\left(\mathrm{I}^{2}=0 \%, \mathrm{p}=0.51\right)$ (Fig. 2, Table 3 ). There was an asymmetry of the points on visual estimation of the funnel plot, which indicated the possibility of publication bias with respect to the primary outcome (all-cause death) (Fig. 3).

\section{Nonfatal myocardial infarction}

The RCT subgroup analysis demonstrated no difference in MI incidence (10.3\% DES vs. $5.9 \%$ CABG, RR 1.44, 95\% CI 0.79-2.60, $\mathrm{p}=0.23$ ), with a significant between-trial heterogeneity 
Table 3. Sensitivity analysis.

\begin{tabular}{|c|c|c|c|c|c|c|}
\hline & $\begin{array}{c}\text { Outcome } \\
\text { (total subjects) }\end{array}$ & $\begin{array}{l}\text { PCI-DES } \\
(\%)\end{array}$ & $\begin{array}{c}\text { CABG } \\
(\%)\end{array}$ & $\begin{array}{l}\text { RR } 95 \% \\
\text { [CI] }\end{array}$ & $\mathbf{P}$ & Heterogeneity \\
\hline $\begin{array}{l}\text { OCT analysis after } \\
\text { excluding: } \\
\text { Javaid, } 2007 \text { trial }\end{array}$ & $\begin{array}{c}\text { Death } \\
(n=4,691)\end{array}$ & $\begin{array}{c}231 / 2,341 \\
(9.9 \%)\end{array}$ & $\begin{array}{c}223 / 2,350 \\
(9.5 \%)\end{array}$ & $\begin{array}{c}1.02 \\
{[0.86-1.22]}\end{array}$ & 0.80 & $\begin{array}{l}I^{2}=0 \% \\
P=0.73\end{array}$ \\
\hline $\begin{array}{l}\text { RCT analysis after } \\
\text { excluding: }\end{array}$ & $\begin{array}{c}\text { Death } \\
(n=2,854)\end{array}$ & $\begin{array}{l}195 / 1,438 \\
(13.56 \%)\end{array}$ & $\begin{array}{c}141 / 1,416 \\
(9.96 \%)\end{array}$ & $\begin{array}{c}1.36 \\
{[1.11-1.66]}\end{array}$ & 0.003 & $\begin{array}{l}I^{2}=0 \% \\
P=0.53\end{array}$ \\
\hline \multirow[t]{2}{*}{ VA CARDS trial } & $\begin{array}{l}\text { Myocardial } \\
\text { infarction } \\
(n=2,854)\end{array}$ & $\begin{array}{l}153 / 1,438 \\
(10.64 \%)\end{array}$ & $\begin{array}{l}75 / 1,416 \\
(5.30 \%)\end{array}$ & $\begin{array}{c}2.01 \\
{[1.54-2.62]}\end{array}$ & $<0.00001$ & $\begin{array}{l}P^{2}=0 \% \\
P=0.83\end{array}$ \\
\hline & $\begin{array}{l}\text { Repeat } \\
\text { revascularization } \\
(\mathrm{n}=2,854)\end{array}$ & $\begin{array}{l}249 / 1,438 \\
(17.32 \%)\end{array}$ & $\begin{array}{l}93 / 1,416 \\
(6.57 \%)\end{array}$ & $\begin{array}{c}2.61 \\
{[2.09-3.27]}\end{array}$ & $<0.00001$ & $\begin{array}{l}I^{2}=0 \% \\
P=0.89\end{array}$ \\
\hline \multirow{3}{*}{$\begin{array}{l}\text { Overall analysis } \\
\text { after excluding: } \\
\text { VA CARDS trial }\end{array}$} & $\begin{array}{c}\text { Death } \\
(\mathrm{n}=7,545) \#\end{array}$ & $\begin{array}{l}426 / 3,779 \\
(11.27 \%)\end{array}$ & $\begin{array}{c}364 / 3,766 \\
(9.67 \%)\end{array}$ & $\begin{array}{c}1.15 \\
{[1.01-1.31]}\end{array}$ & 0.04 & $\begin{array}{l}I^{2}=0 \% \\
P=0.51\end{array}$ \\
\hline & $\begin{array}{l}\text { Myocardial } \\
\text { infarction } \\
(n=7,467)\end{array}$ & $\begin{array}{c}326 / 3,786 \\
(8.61 \%)\end{array}$ & $\begin{array}{c}159 / 3,681 \\
(4.32 \%)\end{array}$ & $\begin{array}{c}1.91 \\
{[1.43-2.57]}\end{array}$ & $<0.0001$ & $\begin{array}{l}I^{2}=43 \% \\
P=0.04\end{array}$ \\
\hline & $\begin{array}{l}\text { Repeat } \\
\text { revascularization } \\
(\mathrm{n}=8,643)\end{array}$ & $\begin{array}{l}834 / 4,394 \\
(18.98 \%)\end{array}$ & $\begin{array}{c}255 / 4,249 \\
(6.00 \%)\end{array}$ & $\begin{array}{c}2.99 \\
{[2.62-3.42]}\end{array}$ & $<0.00001$ & $\begin{array}{l}I^{2}=0 \% \\
P=0.57\end{array}$ \\
\hline
\end{tabular}

\#Excluding VA CARDS trial and Javaid, 2007 trial; RCTs — randomized controlled trials; OCTs — observational controlled trial; RR — risk ratio; $\mathrm{Cl}$ - confidence interval; PCI-DES - percutaneous coronary intervention with drug-eluting stents; CABG - coronary artery bypass grafting

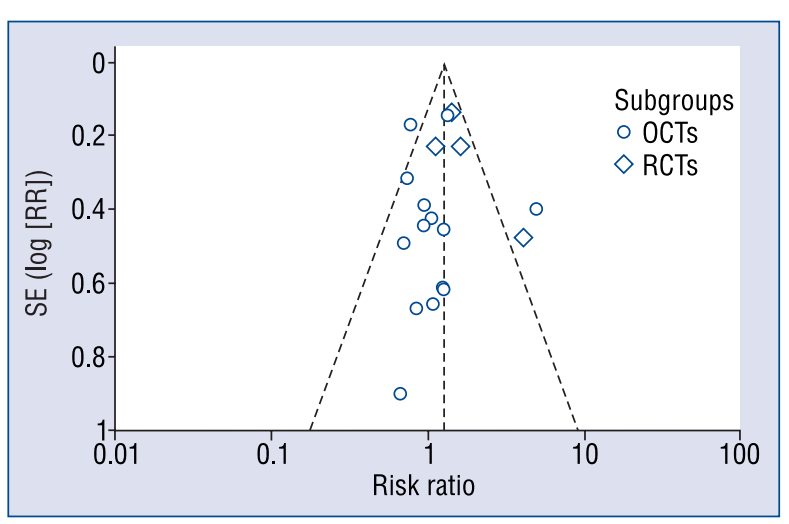

Figure 3. Funnel plot for assessment of publication bias of trials for the endpoint of all-cause death; OCTs observational controlled trial; RCTs - randomized controlled trials.

$\left(\mathrm{I}^{2}=75 \%, \mathrm{p}=0.007\right)$. Sensitivity analysis showed that this heterogeneity was largely contributed by the inclusion of the VA CARDS trial, where MI rate after $\mathrm{CABG}$ was much higher than that after PCI-DES, unlike other RCTs. When excluding this trial, random-effects meta-analysis demonstrated a statistical difference (10.6\% DES vs. $5.3 \%$ CABG, RR 2.01, 95\% CI 1.54-2.62, $\mathrm{p}<0.00001)$ with no residual heterogeneity $\left(\mathrm{I}^{2}=0 \%, \mathrm{p}=0.83\right)$. The OCT subgroup analysis showed that MI after PCI-DES was more prevalent as opposed to CABG $(7.4 \%$ DES vs. 3.7\% CABG, RR 1.82, 95\% CI 1.15-2.86, $\mathrm{p}=0.01)$ with a moderate heterogeneity $\left(\mathrm{I}^{2}=54 \%\right.$, $\mathrm{p}=0.01)$, for which no special trial was mostly responsible.

Regardless of the inclusion or exclusion of the VA CARDS trial, random-effects metaanalysis showed that the overall MI rate was consistently higher in PCI-DES patients (inclusion: 8.5\% DES vs. $4.6 \%$ CABG, RR $1.68,95 \%$ CI 1.20 $-2.37, \mathrm{p}=0.003$; exclusion: $8.6 \%$ DES vs. $4.3 \%$ CABG, RR 1.91, 95\% CI 1.43-2.57, p < 0.0001) (Fig. 4, Table 3).

\section{Nonfatal stroke}

Fixed-effects meta-analysis revealed that both in the RCTs and OCTs subgroup PCI-DES was associated with much lower risk of stroke compared with CABG (RCTs: $2.3 \%$ DES vs. $3.8 \%$ CABG, RR 0.59, 95\% CI 0.39-0.90, $\mathrm{p}=0.01$; OCTs: $1.8 \%$ DES vs. $4.0 \%$ CABG, RR 0.46, 95\% CI 0.32-0.66, $\mathrm{p}<0.0001$ ) with no heterogeneity between trials (RCTs: $\mathrm{I}^{2}=0 \%, \mathrm{p}=0.94$; OCTs: $\mathrm{I}^{2}=0 \%, \mathrm{p}=0.57$ ). When pooling two subgroups, PCI-DES patients kept a consistently lower frequency of stroke $(2.0 \%$ DES vs. 3.9\% CABG, RR 0.51, 95\% CI 0.39-0.67, $\mathrm{p}<0.00001)$ with no heterogeneity between trials $\left(\mathrm{I}^{2}=0 \%, \mathrm{p}=0.80\right)$ (Fig. 5).

\section{Repeat revascularization}

The RCT subgroup analysis showed that patients after PCI-DES risk a several-fold higher 


\begin{tabular}{|c|c|c|c|c|c|c|c|c|}
\hline \multirow{2}{*}{$\frac{\text { Study or Subgroup }}{1.4 .1 \text { OCTs }}$} & \multicolumn{2}{|c|}{ PCI-DES } & Events & CABG & Weight & $\begin{array}{c}\text { Risk Ratio } \\
\text { M-H, Random. } 95 \% \mathrm{Cl}\end{array}$ & \multicolumn{2}{|c|}{$\begin{array}{c}\text { Risk Ratio } \\
\text { M-H, Random, } 95 \% \mathrm{Cl}\end{array}$} \\
\hline & & & & Total & & 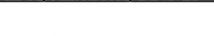 & & \multirow{2}{*}{$\longrightarrow$} \\
\hline Ben-Gal2006 & 25 & 86 & 5 & 86 & $3.2 \%$ & $5.00[2.01,12.45]$ & & \\
\hline Briguori200? & 13 & 69 & 7 & 149 & $3.4 \%$ & $4.01[1.67,9.60]$ & & $\longrightarrow$ \\
\hline Contini2012 & 108 & 359 & 37 & 359 & $10.0 \%$ & $2.92[2.07,4.12]$ & & $\rightarrow$ \\
\hline Domínguez-Franco2008 & 15 & 124 & 6 & 139 & $3.2 \%$ & $2.80[1.12,7.00]$ & & $\cdot$ \\
\hline Javaid 2007 & 54 & 344 & 11 & 257 & $5.5 \%$ & $3.67[1.96,6.87]$ & & $\rightarrow$ \\
\hline Kim2009 & 28 & 251 & 5 & 256 & $3.1 \%$ & $5.71[2.24,14.56]$ & & $\longrightarrow$ \\
\hline Lee2007 & 20 & 102 & 3 & 103 & $2.1 \%$ & $6.73[2.06,21.96]$ & & \\
\hline Luo2012 & 14 & 99 & 3 & 127 & $2.0 \%$ & $5.99[1.77,20.26]$ & & \\
\hline Moshkovitz2012 & 91 & 271 & 32 & 226 & $9.6 \%$ & $2.37[1.65,3.41]$ & & $\rightarrow$ \\
\hline Onuma2011 & 50 & 159 & 10 & 96 & $5.5 \%$ & $3.02[1.61,5.67]$ & & $\rightarrow$ \\
\hline Park2011 & 91 & 489 & 22 & 402 & $8.0 \%$ & $3.40[2.18,5.31]$ & & $\rightarrow$ \\
\hline Qia02009 & 42 & 363 & 4 & 282 & $2.7 \%$ & $8.16[2.96,22.48]$ & & \\
\hline Tarantini2008 & 7 & 93 & 5 & 127 & $2.3 \%$ & $1.91[0.63,5.84]$ & & \\
\hline Yamagata2010 & 19 & 92 & 8 & 116 & $4.1 \%$ & $2.99[1.37,6.53]$ & & $\longrightarrow$ \\
\hline Zhao2011 & 8 & 55 & 4 & 108 & $2.2 \%$ & $3.93[1.24,12.47]$ & & \\
\hline Subtotal $(95 \% \mathrm{Cl})$ & & 2956 & & 2833 & $66.8 \%$ & $3.22[2.73,3.80]$ & & $\downarrow$ \\
\hline Total events & 585 & & 162 & & & & & \\
\hline \multicolumn{9}{|c|}{ Heterogeneity: $\operatorname{Tau}^{2}=0.00 ; \mathrm{Chi}^{2}=12.94, \mathrm{df}=14(\mathrm{P}=0.53) ; \mathrm{I}^{2}=0 \%$} \\
\hline \multicolumn{9}{|c|}{ Test for overall effect: $Z=13.88(P<0.00001)$} \\
\hline \multicolumn{9}{|l|}{ 1.4.2 RCTs } \\
\hline CARDia & 57 & 254 & 23 & 248 & $8.0 \%$ & $2.42[1.54,3.80]$ & & $\rightarrow$ \\
\hline FREEDOM & 117 & 953 & 42 & 947 & $10.0 \%$ & $2.77[1.97,3.89]$ & & $=$ \\
\hline SYNTAX & 75 & 231 & 28 & 221 & $9.0 \%$ & $2.56[1.73,3.80]$ & & $\rightarrow$ \\
\hline VA CARDS & 19 & 101 & 19 & 97 & $6.2 \%$ & $0.96[0.54,1.70]$ & & \\
\hline Subtotal (95\% Cl) & & 1539 & & 1513 & $33.2 \%$ & $2.11[1.41,3.15]$ & & $>$ \\
\hline Total events & 268 & & 112 & & & & & \\
\hline \multirow{2}{*}{\multicolumn{9}{|c|}{$\begin{array}{l}\text { Heterogeneity: } \text { Tau }^{2}=0.12 ; \mathrm{Chi}^{2}=10.48, \mathrm{df}=3(\mathrm{P}=0.01) ; \mathrm{l}^{2}=71 \% \\
\text { Test for overall effect: } Z=3.65(\mathrm{P}=0.0003)\end{array}$}} \\
\hline & & & & & & & & \\
\hline Total $(95 \% \mathrm{Cl})$ & & 4495 & & 4346 & $100.0 \%$ & $2.95[2.46,3.55]$ & & $\checkmark$ \\
\hline Total events & 853 & & 274 & & & & & \\
\hline \multicolumn{7}{|c|}{ Heterogeneity: $\operatorname{Tau}^{2}=0.06 ; \mathrm{Chi}^{2}=29.94, \mathrm{df}^{2}=18(\mathrm{P}=0.04) ; \mathrm{l}^{2}=40 \%$} & $\begin{array}{lll}0.01 & 0.1 & 1\end{array}$ & $10 \quad 100$ \\
\hline \multicolumn{7}{|c|}{$\begin{array}{l}\text { Test for overall effect: } Z=11.54(P=0.00001) \\
\text { Test for subaroun differences: } \mathrm{Chi}^{2}=3.68 \mathrm{df}=1(\mathrm{P}=0.06) . \mathrm{I}^{2}=72.8 \%\end{array}$} & Favours PCl-DES $F$ & Favours $C A B G$ \\
\hline
\end{tabular}

Figure 6. Repeat revascularization at longest follow-up. The individual trials and subtotal risk ratios and $95 \%$ confidence intervals $(\mathrm{Cl})$ comparing the outcome of repeat revascularization for percutaneous coronary intervention with drug-eluting stents (PCI-DES) vs. coronary artery bypass grafting (CABG).

rate of subsequent revascularization $(17.4 \%$ DES vs. $7.4 \%$ CABG, RR 2.11, 95\% CI 1.41-3.15, $\mathrm{p}=0.0003$ ), even if including the VA CARDS trial which primarily contributed to the significant heterogeneity among RCTs $\left(\mathrm{I}^{2}=71 \%, \mathrm{p}=0.01\right)$. After exclusion of this trial, the point estimate for repeat revascularization reached a statistical significance (17.32\% DES vs. $6.57 \%$ CABG, RR 2.61, 95\% CI 2.09-3.27, $\mathrm{p}<0.00001$ ) with no residual heterogeneity $\left(\mathrm{I}^{2}=0 \%, \mathrm{p}=0.89\right)$. The OCT subgroup analysis suggested patients after PCI-DES was at a substantially higher risk of repeat revascularization (19.8\% DES vs. 5.7\% CABG, RR 3.22, 95\% CI $2.73-3.80, \mathrm{p}=0.0001)$ with no heterogeneity among OCTs $\left(\mathrm{I}^{2}=0 \%, \mathrm{p}=0.53\right)$.

Random-effects meta-analysis showed that the overall incidence of subsequent revascularization was consistently much higher in PCI-DES cohort regardless of the inclusion or exclusion of the VA CARDS trial (inclusion: $19.0 \%$ DES vs. $6.3 \%$ CABG, RR 2.95, 95\% CI 2.46-3.55, p < 0.00001; exclusion: $19.0 \%$ DES vs. $6.0 \%$ CABG, RR 2.99, $95 \%$ CI 2.62-3.42, p < 0.0001) (Fig. 6, Table 3).

\section{Discussion}

To the best of our knowledge, this study, incorporating more than 8,000 patients, is the first systematic review and meta-analysis of both the randomized and nonrandomized studies that compared PCI-DES vs. CABG in diabetic subset with LM and/or MVD. Not only did this meta-analysis conclude the overall efficacy and safety of the two revascularization strategies in the general population, but also compared the two strategies in real-world patients (OCTs) and in high-selected patients (RCTs), respectively.

It is well known that the RCTs are the most convincing in all types of studies, while the OCTs, unlike the RCTs which enroll the high-selected subjects, can reflect daily clinical practice where the choice between two revascularization strategies mostly depends on the clinician's discretion and the patient's will. Actually, compared with nondiabetic patients, diabetics risk higher mortality after either DES or CABG, which demonstrated that neither CABG nor PCI could eliminate the 
increased mortality risk conferred by diabetes mellitus [40]. However, CABG was generally considered a preferred revascularization strategy for patients with LM and/or MVD [9]. In the daily practice, the clinicians preferred CABG for the patients with more complex lesions regardless of diabetes mellitus status, whereas patients who have local diseases and less extensive lesions were more likely to undergo DES. Thus, in the OCTs, the CABG cohorts had higher-risk profiles for clinical and angiographic characteristics, which were revealed by the higher EuroSCORE and/or higher SYNTAX score, and by the higher prevalence of triple and/or left main CAD in the CABG cohort (Table 2).

Several previous meta-analyses comparing PCI-DES vs. CABG for diabetics showed that no significant differences in death and MI were found, but the lower risk of repeat revascularization and the higher risk of stroke in CABG patients had been definitely revealed [41, 42]. The FREEDOM trial was the biggest prospective RCT comparing the two revascularization modalities for diabetic patients with MVD exclusively. The significantly higher mortality and MI but the lower rate of stroke in the PCI-DES group was observed actually at its 5 -year follow-up, even though this trial was underpowered to detect a difference in all-cause mortality [21]. However, the debate had not been discontinued. Subsequently, the SYNTAX trial compared CABG with PCI using paclitaxel-eluting stents (PCI-PES) for patients with LM and/or three-vessel disease, and its subgroup analysis for the diabetic subset revealed that the mortalities of two strategies were not statistically different (19.5\% DES vs. $12.9 \%$ CABG, $p=0.065)$, but this analysis was also underpowered and limited by being post hoc exploratory subgroup analysis $[20,43]$. The Hakeem et al. trial [44], the first meta-analysis of RCTs on this topic, revealed that CABG improved survival, decreased $\mathrm{MI}$ and repeat revascularization, but increased the risk of stroke compared with multivessel PCI with first-generation DES. Generally, it was definite that patients after CABG patients risked more strokes and less repeat revascularization compared with PCI-DES patients. Through the sensitivity analyses and the subgroup analyses (the same results from two subgroup analyses), this meta-analysis further confirmed that CABG had significant advantage in repeat revascularization but the substantial disadvantage in stroke.

The funnel plot showed that the studies were mainly scattered left of the line, which revealed that publication bias possibly existed and the advantage of CABG in all-cause death was likely underestimated (Fig. 3). However, the RCT subgroup analysis and the overall sensitivity analysis demonstrated that CABG had an advantage over PCI-DES with the respects of all-cause death. The RCT subgroup analysis showed that CABG improved survival compared with PCI-DES, while the OCT subgroup analysis demonstrated the comparable mortality between two strategies (Fig. 2, Table 3). The different outcomes between the OCT and RCT subgroup analysis were likely to be driven by the higher-risk profiles for clinical and angiographic characteristics in $\mathrm{CABG}$ cohort in the OCTs, while the RCTs had comparable risk factor between two cohorts (Table 2), which was possibly another reason for the asymmetry of the funnel plot. The comparable survival between two strategies in OCT subgroup and the lower mortality of the OCT patients than RCT patients $(9.6 \%$ OCTs vs. $11.9 \%$ RCTs, RR $0.81,95 \%$ CI $0.71-0.92, \mathrm{p}=0.001$ ) suggested that the preferred choice of CABG for diabetic patients with higherrisk profiles in daily clinical practice was reasonable in terms of all-cause death. Conclusively, CABG had advantage over PCI-DES with respect of the primary endpoint despite the different results between RCTs and OCTs subgroup analyses.

Only the VA CARDS trial conducted the aggressive search for silent MI, while other RCTs included silent MI when discovered but not mandated serial electrocardiography and nuclear studies, which was one of the reasons that the MI rate following CABG was much higher than that after PCI-DES [19], which pronouncedly differed from other RCTs. However, regardless of inclusion or exclusion of the VA CARDS trial, our study confirmed consistently that PCI-DES was associated with higher MI rate compared with CABG. The CABG advantage over PCI-DES in MI was also revealed by the Lee et al. [42] trial which was a meta-analysis including OCTs only and by the Hakeem et al. trial [44] which was a meta-analysis including RCTs only. Generally, the advantage of CABG in MI was definite, but it was unknown whether this advantage was kept when silent MI was aggressively searched for.

\section{Limitations of the study}

We acknowledged that our study had several limitations. Firstly, the observational studies enrolled in this mate-analysis had their inherent limitations, namely design bias, selection bias, treatment bias and publication bias. Substantial between-studies heterogeneity in terms of both study design and the effect sizes of each end point 
were observed in this review. Furthermore, this meta-analysis contained trials regardless of the baseline characteristics of patients, medication treatment after revascularization, duration of follow-up and location of occlusion, which might bring about more heterogeneity. Thus, despite our choice of random effects models, the incorporating of disparate studies could not accurately summarize the overall effect of two strategies, and also limits the generalizability of our results. Secondly, the first-generation DES, used mostly in the included studies, could not fully reflect the clinical practice in the newer-generation DES era. Recent data from a Swedish registry, enrolling 4,751 diabetic patients, showed that the use of the second-generation stents (everolimus-eluting stent) was associated with improved outcomes compared with the firstgeneration (PES and sirolimus-eluting stents), but the result was mainly driven by lower rates of stent thrombosis and death [45]. Whether CABG keeps the priority to the PCI-DES is unknown. Thirdly, several trials reporting the in-hospital and 30-day follow-up result showed that CABG was associated with increased major adverse cardiac events, death, stroke. The meta-analysis for these short-term endpoints was not conducted by our study, which makes it difficult to evaluate the efficacy and safety of the two strategies entirely.

\section{Conclusions}

CABG for patients with diabetes mellitus and LM and/or MVD had advantages over PCI-DES in all-cause death, nonfatal MI, and repeat revascularization, but one substantial disadvantage in nonfatal stroke. The high-selected patients (RCTs) risked a higher mortality than the real-world patients (OCTs).

\section{Conflict of interest: None declared}

\section{References}

1. Hammoud T, Tanguay J, Bourassa MG. Management of coronary artery disease: Therapeutic options in patients with diabetes. J Am Coll Cardiol, 2000; 36: 355-365.

2. Kannel WB, McGee DL. Diabetes and cardiovascular disease. JAMA, 1979; 241: 2035-2038.

3. Kip KE, Faxon DP, Detre KM, Yeh W, Kelsey SF, Currier JW. Coronary angioplasty in diabetic patients the National Heart, Lung, and Blood Institute percutaneous transluminal coronary angioplasty registry. Circulation, 1996; 94: 1818-1825.

4. Stein B, Weintraub WS, Gebhart SS, et al. Influence of diabetes mellitus on early and late outcome after percutaneous transluminal coronary angioplasty. Circulation, 1995; 91: 979-989.

5. Murray CJ, Lopez AD. Global mortality, disability, and the contribution of risk factors: Global Burden of Disease Study. Lancet, 1997; 349: 1436-1442.
6. Mathew V, Gersh BJ, Williams BA et al. Outcomes in patients with diabetes mellitus undergoing percutaneous coronary intervention in the current era: A report from the prevention of restenosis with tranilast and its outcomes (PRESTO) trial. Circulation, 2004; 109: 476-480.

7. Suero JA, Marso SP, Jones PG et al. Procedural outcomes and long-term survival among patients undergoing percutaneous coronary intervention of a chronic total occlusion in native coronary arteries: A 20-year experience. J Am Coll Cardiol, 2001; 38: 409-414.

8. Elezi S, Kastrati A, Pache J et al. Diabetes mellitus and the clinical and angiographic outcome after coronary stent placement. J Am Coll Cardiol, 1998; 32: 1866-1873.

9. Wijns W, Kolh P, Danchin N et al. Guidelines on myocardial revascularization The Task Force on Myocardial Revascularization of the European Society of Cardiology (ESC) and the European Association for Cardio-Thoracic Surgery (EACTS). Eur Heart J, 2010; 31: 2501-2555.

10. Morice M, Serruys PW, Sousa JE et al. A randomized comparison of a sirolimus-eluting stent with a standard stent for coronary revascularization. New Engl J Med, 2002; 346: 1773-1780.

11. Moses JW, Leon MB, Popma JJ et al. Sirolimus-eluting stents versus standard stents in patients with stenosis in a native coronary artery. New Engl J Med, 2003; 349: 1315-1323.

12. Stone GW, Ellis SG, Cox DA et al. A polymer-based, paclitaxeleluting stent in patients with coronary artery disease. New Engl J Med, 2004; 350: 221-231.

13. Grube E, Silber S, Hauptmann KE et al. TAXUS I six-and twelvemonth results from a randomized, double-blind trial on a slow-release paclitaxel-eluting stent for de novo coronary lesions. Circulation, 2003; 107: 38-42.

14. Colombo A, Drzewiecki J, Banning A et al. Randomized study to assess the effectiveness of slow- and moderate-release polymerbased paclitaxel-eluting stents for coronary artery lesions. Circulation, 2003; 108: 788-794.

15. Babapulle MN, Joseph L, Bélisle P, Brophy JM, Eisenberg MJ. A hierarchical Bayesian meta-analysis of randomised clinical trials of drug-eluting stents. Lancet, 2004; 364: 583-591.

16. Hermiller JB, Raizner A, Cannon L et al. Outcomes with the polymer-based paclitaxel-eluting Taxus stent in patients with diabetes mellitusthe TAXUS-IV trial. J Am Coll Cardiol, 2005; 45: 1172-1179.

17. Moussa I, Leon MB, Baim DS et al. Impact of Sirolimus-Eluting Stents on Outcome in Diabetic Patients A SIRIUS (SIRolImUScoated Bx Velocity balloon-expandable stent in the treatment of patients with de novo coronary artery lesions) Substudy. Circulation, 2004; 109: 2273-2278.

18. Hoffman SN, TenBrook JA, Wolf MP, Pauker SG, Salem DN, Wong JB. A meta-analysis of randomized controlled trials comparing coronary artery bypass graft with percutaneous transluminal coronary angioplasty: One-to eight-year outcomes. J Am Coll Cardiol, 2003; 41: 1293-1304.

19. Kamalesh M, Sharp TG, Tang XC, et al. Percutaneous coronary intervention versus coronary bypass surgery in United States veterans with diabetes. [Journal Article; Multicenter Study; Randomized Controlled Trial; Research Support, U.S. Gov't, Non-P.H.S.]. J Am Coll Cardiol, 2013; 61: 808-816.

20. Kappetein AP, Head SJ, Morice MC et al. Treatment of complex coronary artery disease in patients with diabetes: 5-year results comparing outcomes of bypass surgery and percutaneous coronary intervention in the SYNTAX trial. [Journal Article; Research Support, Non-U.S. Gov't]. Eur J Cardiothorac Surg, 2013; 43: 1006-1013. 
21. Farkouh ME, Domanski M, Sleeper LA et al. Strategies for multivessel revascularization in patients with diabetes. [Journal Article; Multicenter Study; Randomized Controlled Trial; Research Support, N.I.H., Extramural; Research Support, Non-U.S. Gov't]. N Engl J Med, 2012; 367: 2375-2384.

22. Kapur A, Hall RJ, Malik IS et al. Randomized comparison of percutaneous coronary intervention with coronary artery bypass grafting in diabetic patients. 1-year results of the CARDia (Coronary Artery Revascularization in Diabetes) trial. [Comparative Study; Journal Article; Randomized Controlled Trial; Research Support, Non-U.S. Gov't]. J Am Coll Cardiol, 2010; 55: 432-440.

23. Ben-Gal Y, Moshkovitz Y, Nesher N et al. Drug-eluting stents versus coronary artery bypass grafting in patients with diabetes mellitus. [Comparative Study; Journal Article; Retracted Publication]. Ann Thorac Surg, 2006; 82: 1692-1697.

24. Briguori C, Condorelli G, Airoldi F et al. Comparison of coronary drug-eluting stents versus coronary artery bypass grafting in patients with diabetes mellitus. [Comparative Study; Journal Article; Research Support, Non-U.S. Gov't]. Am J Cardiol, 2007; 99: 779-784.

25. Contini GA, Nicolini F, Fortuna D et al. Five-year outcomes of surgical or percutaneous myocardial revascularization in diabetic patients. Int J Cardiol, 2013; 168: 1028-1033.

26. Dominguez-Franco AJ, Jimenez-Navarro MF, Hernandez-Garcia JM et al. Comparison of medium-term outcomes obtained with drug-eluting stents and coronary artery bypass grafts in an unselected population of diabetic patients with multivessel coronary disease. Propensity score analysis. [Comparative Study; Journal Article]. Rev Esp Cardiol, 2009; 62: 491-500.

27. Javaid A, Steinberg DH, Buch AN et al. Outcomes of coronary artery bypass grafting versus percutaneous coronary intervention with drug-eluting stents for patients with multivessel coronary artery disease. [Comparative Study; Journal Article]. Circulation, 2007; 116 (11 Suppl.): I200-I1206.

28. Kim WJ, Park DW, Yun SC et al. Impact of diabetes mellitus on the treatment effect of percutaneous or surgical revascularization for patients with unprotected left main coronary artery disease: A subgroup analysis of the MAIN-COMPARE study. [Comparative Study; Journal Article; Multicenter Study; Research Support, Non-U.S. Gov't]. J Am Coll Cardiol Cardiovasc Interv, 2009; 2: 956-963.

29. Lee MS, Jamal F, Kedia G et al. Comparison of bypass surgery with drug-eluting stents for diabetic patients with multivessel disease. [Comparative Study; Journal Article]. Int J Cardiol, 2007; 123: 34-42.

30. Luo Y, Yu X, Chen F et al. Impact of diabetes mellitus on patients with unprotected left main coronary artery lesion disease treated with either percutaneous coronary intervention or coronaryartery bypass grafting. Coron Artery Dis, 2012; 23: 322-329.

31. Moshkovitz Y, Mohr R, Medalion B et al. Drug-eluting stents compared with bilateral internal thoracic artery grafts for diabetic patients. [Comparative Study; Journal Article]. Ann Thorac Surg, 2012; 94: 1455-1462.

32. Onuma Y, Wykrzykowska JJ, Garg S, Vranckx P, Serruys PW. Five-year follow-up of coronary revascularization in diabetic patients with multivessel coronary artery disease: Insights from ARTS (arterial revascularization therapy study)-II and ARTS-I trials. [Clinical Trial; Comparative Study; Journal Article; Multicenter Study]. J Am Coll Cardiol Cardiovasc Interv, 2011; 4: 317-323.

33. Kim YG, Park DW, Lee WS et al. Influence of diabetes mellitus on long-term (five-year) outcomes of drug-eluting stents and coronary artery bypass grafting for multivessel coronary revascularization. [Journal Article; Research Support, Non-U.S. Gov't]. Am J Cardiol, 2012; 109: 1548-1557.

34. Park DW, Kim YH, Song HG et al. Long-term comparison of drug-eluting stents and coronary artery bypass grafting for multivessel coronary revascularization: 5-year outcomes from the Asan Medical Center-Multivessel Revascularization Registry. [Comparative Study; Journal Article; Research Support, NonU.S. Gov't]. J Am Coll Cardiol, 2011; 57: 128-137.

35. Qiao Y, Ma C, Nie S et al. Twelve months clinical outcome of drug-eluting stents implantation or coronary artery bypass surgery for the treatment of diabetic patients with multivessel disease. [Comparative Study; Journal Article]. Clin Cardiol, 2009; 32: E24-E30.

36. Tarantini G, Ramondo A, Napodano M et al. PCI versus CABG for multivessel coronary disease in diabetics. [Comparative Study; Journal Article]. Catheter Cardiovasc Interv, 2009; 73: 50-58.

37. Yamagata K, Kataoka Y, Kokubu N et al. A 3-year clinical outcome after percutaneous coronary intervention using sirolimus-eluting stent and off-pump coronary artery bypass grafting for the treatment of diabetic patients with multivessel disease. [Comparative Study; Journal Article]. Circ J, 2010; 74: 671-678.

38. Zhao X, Zhou Y, Song H et al. Comparison of bypass surgery with drug-eluting stents in diabetic patients with left main coronary stenosis. [Journal Article; Research Support, Non-U.S. Gov't]. Yonsei Med J, 2011; 52: 923-932.

39. Higgins JP, Thompson SG, Deeks JJ, Altman DG. Measuring inconsistency in meta-analyses. Br Med J, 2003; 327: 557.

40. Garcia MJ, McNamara PM, Gordon T, Kannell WB. Morbidity and mortality in diabetics in the Framingham population: sixteen year follow-up study. Diabetes, 1974; 23: 105-111.

41. Gao F, Zhou YJ, Shen H, Wang ZJ, Yang SW, Liu XL. Meta-analysis of percutaneous coronary intervention versus coronary artery bypass graft surgery in patients with diabetes and left main and/ /or multivessel coronary artery disease. Acta Diabetol, 2013; 50: 765-773.

42. Lee MS, Yang T, Dhoot J, Iqbal Z, Liao H. Meta-analysis of studies comparing coronary artery bypass grafting with drug-eluting stenting in patients with diabetes mellitus and multivessel coronary artery disease. [Journal Article; Meta-Analysis]. Am J Cardiol, 2010; 105: 1540-1544.

43. Mohr FW, Morice MC, Kappetein AP et al. Coronary artery bypass graft surgery versus percutaneous coronary intervention in patients with three-vessel disease and left main coronary disease: 5-year follow-up of the randomised, clinical SYNTAX trial. [Journal Article; Multicenter Study; Randomized Controlled Trial; Research Support, Non-U.S. Gov't]. Lancet, 2013; 381: 629-638.

44. Hakeem A, Garg N, Bhatti S, Rajpurohit N, Ahmed Z, Uretsky BF. Effectiveness of percutaneous coronary intervention with drug-eluting stents compared with bypass surgery in diabetics with multivessel coronary disease: Comprehensive systematic review and meta-analysis of randomized clinical data. J Am Heart Assoc, 2013; 2: e354.

45. Kedhi E, Gomes ME, Lagerqvist B et al. Clinical impact of second-generation everolimus-eluting stent compared with firstgeneration drug-eluting stents in diabetes mellitus patients: Insights from a nationwide coronary intervention register. [Comparative Study; Journal Article]. J Am Coll Cardiol Cardiovasc Interv, 2012; 5: 1141-1149. 